\title{
Laparoscopic approach to pelvic organ prolapse - the way to go or a blind alley?
}

\author{
Ewelina Malanowska ${ }^{1}$, Marek Soltes ${ }^{2}$, Andrzej Starczewski ${ }^{1}$, Eckhard Petri $^{3}$, Marcin Jozwik ${ }^{4}$ \\ ${ }^{1}$ Department of Gynecology, Endocrinology and Gynecologic Oncology, Pomeranian Medical University, Szczecin, Poland \\ ${ }^{2} 1^{\text {st }}$ Department of Surgery, Pavol Jozef Safarik University, Kosice, Slovak Republic \\ ${ }^{3}$ Department of Obstetrics and Gynecology, University of Greifswald, Greifswald, Germany \\ ${ }^{4}$ Department of Gynecology and Obstetrics, University of Warmia and Mazury, Olsztyn, Poland
}

Videosurgery Miniinv 2019; 14 (4): 469-475

DOI: https://doi.org/10.5114/wiitm.2019.88749

\begin{abstract}
Pelvic organ prolapse represents a relatively frequent diagnosis that requires attention due to its detrimental effect on quality of life. Not surprisingly, it is one of the commonest indications for surgery in premenopausal and postmenopausal women, often requiring a complex multidisciplinary approach. Traditional vaginal procedures are being gradually replaced by laparoscopic techniques, offering anticipated benefits in reduced recurrence and complication rates, while respecting the trend towards uterus sparing if desirable. Recently, questions about the safety of alloplastic materials used in pelvic organ prolapse surgery were raised, leading to official restrictions in their use, particularly for transvaginal application. As a result, laparoscopic procedures might appear slightly favored but caution must be taken to assure proper technique of mesh placement while maintaining high awareness of possible long-term mesh-related complications that require close surveillance. Therefore, adequate education and training becomes even more important to achieve optimal results and to avoid possible serious medico-legal charges.
\end{abstract}

Key words: complications, laparoscopy, laparoscopic surgery, mesh, pelvic organ prolapse.

\section{Introduction}

Pelvic organ prolapse is defined as a descent of pelvic organs associated with dysfunction of urethral or rectal continence, resulting from defective pelvic support and increased intra-abdominal pressure. The clinical presentation includes prolapse of the vagina and/or uterus and other pelvic organs, typically the urinary bladder, less often the rectum, which makes pelvic organ prolapse a very complex multidisciplinary issue. Risk factors for this condition include menopause, hypoestrogenism, history of vaginal delivery, obesity, connective tissue disorders, chronic cough, constipation and genetic predisposition [1].
Prevalence of pelvic organ prolapse reported in the literature is variable. Depending on the target population and study methodology, it ranges from 3\% to $6 \%$ if the diagnosis is based solely on clinical presentation, to $50 \%$ when pelvic examination is used as a diagnostic measure [2]. Having a detrimental effect on the quality of life, pelvic organ prolapse represents one of the most common indications for surgery in premenopausal and postmenopausal women [3].

The degree of pelvic floor dysfunction can be evaluated objectively using the Pelvic Organ Prolapse Quantification (POP-Q) system developed by the International Continence Society (ICS). This scale describes the degree and location of the prolapse more accurately than other available instruments

\footnotetext{
Address for correspondence

Assoc. Prof. Marek Soltes MD, PhD, $1^{\text {st }}$ Department of Surgery, Pavol Jozef Safarik University, Tr. SNP 1, 04190 Kosice, Slovak Republic, e-mail: soltes.marek@yahoo.com
} 
and is the most widespread measure of pelvic floor dysfunction used worldwide [4].

In 1994, De Lancey proposed a classification of pelvic organ prolapse disorders based on the level they occurred. According to this classification, level I defects include injuries of uterosacral ligaments, enterocele, and prolapse of the uterus, cervix or vaginal vault (apical defect), level II defects include injuries of the vesicovaginal or rectovaginal fascia, cystocele and rectocele, and level III defects include injuries of urethral supporting ligaments and perineum, and ureterocele [5].

Anterior defects found at level II can be further subdivided according to the localization of injury to the vesicovaginal fascia into central, which results in the formation of smooth cystocele without vaginal rugae, and lateral, characterized by the presence of visible rugae within the cystocele. Distinguishing between these two conditions is crucial for the choice of future reconstructive treatment [6].

In recent years, reconstructive surgery has become a dominant treatment strategy for pelvic floor dysfunction. Reconstructive procedures, aimed at the restoration of normal anatomy and function of the pelvic floor, may involve the patient's tissues or synthetic materials. Surgical treatment can only be considered recommended if pelvic organ prolapse leads to clinical symptoms, such as urinary retention, recurrent urinary tract infection, constipation, discomfort and sexual problems [2]. Noticeably, more than half of multigravidas present with POP-Q grade 2 disorders, frequently asymptomatic [7]. In such cases, the decision about surgical treatment, if any, should be made with particular caution [8]. Also of note, no consensus has been reached with regards to the clear definition of "normal" and "abnormal" pelvic floor anatomy. As such, indication for urogynecological surgery is still based on individual patient assessment.

Choice of surgical procedure must respect the anatomical type of pelvic floor dysfunction. Besides traditional vaginal surgeries, laparoscopic repair of the pelvic floor is gaining growing attention, primarily due to its lesser invasiveness and high effectiveness resulting from easier access to pelvic support structures [9].

\section{Aim}

This paper aims to review and summarize current issues regarding surgical treatment of pelvic organ prolapse with special focus on laparoscopic procedures.

\section{Mesh repair - yes or no?}

Several synthetic materials have been proposed and used in urogynecology. Polypropylene, which has more than a 60-year history of successful application in general surgery [10], seems to be the most prevalent one. Another increasingly used option is polyvinylidene fluoride (PVDF), which was shown to have excellent biocompatibility, low risk of bacterial colonization and superior tensile strength maintenance $[11,12]$. Besides the material used, there are also other important parameters that define biocompatibility of the synthetic prosthesis used. These include absorbability, porosity, characteristics of the filament, type of knitting and weight. Porosity seems to be the key issue as microporous meshes are subject to encapsulation, while macroporous ones (pore diameter greater than $75 \mu \mathrm{m}$ ) are easily infiltrated by macrophages and fibroblasts and also readily overgrown by the components of extracellular matrix, thus leading to desirable incorporation. Also of note, multifilament material is theoretically more prone to infection compared to monofilament. As a result, type I synthetic meshes - macroporous and monofilamentous, currently seem to represent the best choice, although the ideal implant is yet to be found $[13,14]$.

Applicability and safety of synthetic materials in vaginal surgery are still a matter of debate. This is due to the fact that use of synthetic prostheses may be associated with serious complications [15] such as erosion into the vaginal wall, infection, pain, dyspareunia, as well as injuries to the nerves, vessels and adjacent organs, i.e. urinary bladder and rectum, during the mesh insertion. The incidence of vaginal wall erosion is reported as up to $33 \%$, while the risk of this complication increases in older women, patients with estrogen deficiency or history of previous vaginal surgery, diabetics and cigarette smokers [16]. Also of note, it seems that surgical technique and surgeon's experience are as important determinants of outcomes as the type of implanted material [17].

Based on the above, in 2017, the European consensus in Urology and Gynaecology on use of implants for the treatment of vaginal prolapse in perimenopausal women suggests restriction of meshes for recurrent prolapse and "to the surgeons with 
appropriate training, working within the multidisciplinary referral centers" [18]. However, in April 2019, after several years of systematic assessment the U.S. Food and Drug Administration ordered the manufacturers of all remaining surgical mesh products for transvaginal repair of pelvic organ prolapse to stop distributing and selling them in the United States immediately. Nevertheless, this ban does not apply to transvaginal mesh for stress urinary incontinence or meshes placed transabdominally for pelvic organ prolapse $[19,20]$. This is in line with the opinion of the European Commission (EC) and independent Scientific Committee on Emerging and Newly Identified Health Risks (SCENIHR) affiliated with the EC that consider polypropylene and PVDF prostheses safe in urogynecological surgery [17]. Interestingly, several countries, including England, Scotland, Australia and New Zealand, went even further and also banned some specific synthetic meshes designed for use in urogynecology [21].

With nearly complete takeover of alloplastic materials for pelvic floor surgery in the recent years, the above-mentioned restrictions represent a serious medico-legal issue influencing the choice of surgical procedure. Also of note, the general lack of surgical expertise in traditional approaches based on native tissue repair leads to a wide gap in education and training that will probably have to be addressed in future.

Xenografts, such as implants made of pork skin (Pelvicol), may be considered an alternative to synthetic prostheses. Nevertheless, they cannot be generally recommended in the surgical management of pelvic floor dysfunction due to increased risk of infection. Moreover, undergoing gradual degradation, xenografts probably do not provide stable support in a long-term perspective. Due to lack of sufficient evidence from clinical trials regarding the safety of such tissue implants, they should be used with particular caution [22].

\section{Techniques of surgical repair}

Nowadays, pelvic organ prolapse procedures are performed both from the vaginal approach and laparoscopically. Selection of surgical technique depends to a large degree on the treatment indications, but also on other factors, such as patient age, reported ailments, and, most importantly, the personal expertise and preference of the surgeon [23].
Vaginal procedures include fixation of the cervix or vagina to sacrospinous ligaments using Richter's technique with sutures or tape, anterior or posterior vaginal wall repair with patient's tissues or synthetic materials, and perineoplasty.

Originally, Richter's procedure was described as unilateral fixation of the vagina to the right sacrospinous ligament. The principal idea of the technique was to take the apex of the vagina out of the main pressure zone in the center of the pelvis. While unilateral fixation was supposed to prevent the risk of obstructed defecation, choice of the right sacrospinous ligament as the fixation point should eliminate any interference with the rectosigmoid, which might be dilated due to connective tissue laxity in prolapse patients [24]. A number of modifications of Richter's fixation have been proposed, although failing to demonstrate any additional benefit $[25,26]$.

Richter's fixation is performed primarily in patients with level I defects, usually resulting from the previous hysterectomy. It may also be considered an alternative in the elderly, taking into account the short operating time and limited morbidity of the procedure. Another indication for sacrospinous fixation occurs if preservation of fertility or the uterus is desired [27].

The most commonly reported complication of Richter's fixation is injury to the vessels and nerves located in close proximity to the sacrospinous ligament. Not infrequently, inaccurate placement of sutures may result in their rupture and subsequent failure of the procedure, although success rates as high as $>90 \%$ are reported by experienced surgeons [28, 29]. Bilateral sacrospinous ligament fixation, providing symmetry and a dual support point, appears to result in the same frequency of postoperative anatomic cure as unilateral repair [30].

The indication for anterior vaginal wall repair is cystocele resulting from central defect (at level II according to De Lancey) with the severity corresponding to POP-Q grade 2 or higher. Overlooking an apical defect as a cause of cystocele may frequently result in misdiagnosis and inappropriate choice of surgical procedure. Patients in whom isolated defects at level I were misdiagnosed as level II defects will not benefit from the surgical treatment aimed at repair of the latter. This may explain approximately $55 \%$ incidence of recurrent prolapse after anterior vaginal wall repair [31].

In order to avoid recurrence, transvaginal reconstructive procedures with synthetic prostheses have 
been used, especially in patients with cystocele and lateral defects at level II. In these procedures, mesh is inserted into the anterior vaginal wall and its arms are fixed to the obturator foramina and sacrospinous ligaments. Such fixation was shown to be suboptimal, as it may lead to folding of the mesh [32]. Considering the distribution of forces and place of fixation, insertion of mesh into the anterior vaginal wall might not be the best decision in patients with apical defects. In such cases, markedly better outcomes can be achieved with sacropexy, pectopexy or lateral suspension [9].

Posterior vaginal wall repair (colporrhaphy) may be used to repair defects in the posterior compartment. This can be achieved by plication of the patient's native rectovaginal connective tissue (traditional colporrhaphy) or just by plication of individual tears in rectovaginal tissue (site-specific colporrhaphy). Whether posterior vaginal repair is associated with diminished sexual functions and/or dyspareunia is a matter of controversy [33].

In principle, perineoplasty may be indicated to restore De Lancey level III pelvic support. Nevertheless, it needs to be stressed that many authors do not consider perineoplasty a treatment of pelvic floor dysfunction, but merely a reconstructive or cosmetic procedure [34].

Laparoscopic procedures, such as sacropexy, lateral suspension or pectopexy, are gaining growing popularity in the treatment of pelvic organ prolapse. This is partly due to their minimally invasive character resulting in an improved postoperative course and diminished number of wound complications and partly due to pathophysiological considerations regarding apical defects and their reconstruction $[35,36]$.

Currently, the most widely accepted standard in the treatment of apical defects is laparoscopic sacropexy [2]. During this procedure, the uterus (hysterosacropexy), cervix (cervicosacropexy) or vaginal vault (colposacropexy) is fixed to the sacrum (S1-S2) or sacral promontory (promontofixation) with polypropylene tape.

The question of whether amputation of the uterine corpus or hysterectomy should be conducted as part of urogynecological procedures in patients without uterine pathologies is still a matter of ongoing discussion. Based on the published data, amputation of the uterine corpus is frequently performed simultaneously with sacropexy in postmenopausal women, even despite the lack of medical indications for the former [37]. According to many scientific bodies, the decision regarding uterus sparing should be based on the patient preference [2]. Peri- and postmenopausal women are more prone to give their consent to amputation of the uterine corpus, which makes fixation easier for the surgeon but lacks any proven advantage compared to hysterectomy. The emotional "bond to the uterus" constitutes an important psychological issue and seems to affect the perception of a woman's sexual life [38]. This is reflected by the growing number of support organizations, such as Hersfoundation, focused on patients who have experienced problems in sexual life after hysterectomy. Recently, hysterosacropexy, i.e. suspension of the whole uterus, has been gaining growing popularity. In this technique, the uterus is attached to the sacral promontory with a bifurcate flat mesh which runs retroperitoneally, alongside the sacrouterine ligaments. Hysterosacropexy found its indication particularly in young patients, as it spares the uterus and does not negatively affect the quality of sexual life [39]. As such, laparoscopic hysterosacropexy appears to be the optimal treatment option for patients in childbearing age [40].

Laparoscopic sacropexy is considered the gold standard for the repair of apical defects in many centers. However, the procedure is technically fairly demanding and carries a risk of significant complications. Furthermore, the proficiency gain curve in laparoscopic sacropexy is quite flat, the estimated minimum case load being approximately 60 procedures. Also of note, training in advanced laparoscopic techniques imposes time, financial and organizational constraints [41].

The number of randomized clinical trials comparing sacropexy with other surgical techniques used in the treatment of apical defects is limited. Laparoscopic sacropexy is postulated to reconstitute the physiological axis of the vagina, while suspension to sacrospinous ligaments from the vaginal approach moves the vaginal axis posterior with the fixation site located more caudally. Thus, the incidence of recurrent apical defects and/or mesh erosion after laparoscopic sacropexy is slightly lower than after vaginal procedures, even though the results failed to reach statistical significance [9].

At the same time, laparoscopic sacropexy was shown to be associated with higher incidence of de novo cystocele [42]. Moreover, dissection of the tis- 
sues around the sacrum or sacral promontory carries a risk of nerve and vessel injuries. Fixation of the mesh on the left side of the anterior longitudinal ligament may reduce free space for the sigmoid colon, whereas fixation on the right side may be associated with the risk of hypogastric trunk injury. Therefore, defecation disorders represent a well-defined complication after sacropexy. Although often neglected, they may occur in up to $17-37 \%$ of cases [43]. The risk of such complications, however, is dependent to a large extent on the surgeon's experience [44].

Comparison between sacropexy and other surgical procedures can be hindered by the diversity of surgical approach (laparoscopic, robotic, open) and technique (straight mesh tape vs. Y-shaped with extensive dissection of tissues around the vaginal surface corresponding to cystocele or rectocele, type of fixation). The lack of standardization of sacropexy technique markedly limits the possibilities of good quality comparative clinical trials [45].

Laparoscopic lateral suspension represents a valuable alternative to laparoscopic sacropexy. The technique has undergone multiple improvements and modifications since its introduction by Dubuisson in 1998 [46]. During the procedure, T-shaped polypropylene mesh is fixed to the uterine cervix, and then its arms are introduced retroperitoneally towards the lateral abdominal wall, alongside oblique ligaments. Due to the use of a T-shaped mesh, Dubuisson's procedure is suitable for the repair of defects at levels I and II. Reconstitution of physiological vaginal topography at level I contributes to the elevation of the vagina at level II, and thus reconstructive procedures are not needed at this level [47]. Access to the operating field during laparoscopic lateral suspension appears to be much easier compared to sacropexy. This argument supports the indication of Dubuisson's procedure, especially in patients with severe obesity, in whom access to the sacrum may be particularly challenging [48].

On the whole, laparoscopic lateral suspension of the uterus is associated with low incidence of intraoperative complications and recurrent prolapse (approx. 4\%) [46]. Nevertheless, lateral suspension of the cervix or vaginal stub may lead to anterior displacement of the physiological vaginal axis, which may predispose to the occurrence of cystocele and rectocele in the future [49].

In 2007, the technique of laparoscopic pectopexy was described by Noe. During this procedure one end of the mesh is attached to the uterine cervix, whereas its arms are fixed laterally to iliopectineal ligaments (Cooper ligaments) [35]. As a result, the mesh follows broad ligaments and bypasses critical anatomical structures, such as the ureters and intestines. According to Noe, the height of the lateral fixation corresponds to the level of S2, which is enough to restore the physiological vaginal axis. A similar result might be achieved by traditional colposuspension with tension-free approximation of the vaginal wall towards Cooper's ligament [50].

Laparoscopic pectopexy and lateral suspension do not involve the sacral region, and thus do not pose a risk of hypogastric trunk injury and related complications. Also of note, they eliminate the problem of "sacral osteitis" - a rare complication due to fixation of the uterine cervix/vaginal vault to the promontory caused by the fixating material that was introduced too deep, beneath the anterior longitudinal ligament. Further studies are needed to define whether laparoscopic lateral suspension and pectopexy represent a truly better alternative to sacropexy, which seems to be technically more demanding while also associated with greater risk of complications.

\section{Conclusions}

Pelvic organ prolapse is an important medical problem triggering significant socioeconomic costs. It is estimated to affect more than $50 \%$ of perimenopausal women. The primary therapeutic objective is restoration of normal topography that subsequently leads to normalized function of pelvic organs. As such, the surgical strategy has to be based on the location and severity of the pelvic floor defect. Given the etiological variability, a wider range of procedures is required to allow a tailored approach to the individual patient.

Recently, surgical therapy of pelvic organ prolapse has been changing significantly. Traditional vaginal procedures are being replaced by laparoscopic techniques that offer anticipated benefits in reduced recurrence and complication rates while respecting the trend towards uterus sparing, if desirable. Furthermore, safety concerns about alloplastic materials used in pelvic organ prolapse surgery led to their restriction, including a complete ban in some countries, particularly for transvaginal application. Based on the above, the laparoscopic approach to pelvic organ prolapse might appear to be the way to go. 
At the same time, a word of caution should be mentioned here, to avoid ending up in a blind alley. Laparoscopic techniques for pelvic organ prolapse are known to have a relatively long proficiency gain curve. As such, adequate education and training is necessary to achieve optimal results. This is particularly true also due to the currently increased awareness of mesh-related complications. Therefore, proper technique of mesh placement and close surveillance of its long-term consequences must be considered a necessity.

\section{Conflict of interest}

\section{The authors declare no conflict of interest.}

\section{References}

1. Chow D, Rodríguez LV. Epidemiology and prevalence of pelvic organ prolapse. Curr Opin Urol 2013; 23: 293-8.

2. Barber MD, Maher C. Epidemiology and outcome assessment of pelvic organ prolapse. Int Urogynecol I 2013; 24: 1783-90.

3. Barber MD. Pelvic organ prolapse. BMJ 2016; 354: 13853.

4. Persu C, Chapple CR, Cauni V, et al. Pelvic Organ Prolapse quantification system (POP-Q) - a new era in pelvic prolapse staging. Med Life J 2011; 4: 75-81.

5. DeLancey JO. Anatomy and biomechanics of genital prolapse. Clin Obstet Gynecol 1993; 36: 897-909.

6. Geynisman-Tan J, Kenton K. Surgical updates in the treatment of pelvic organ prolapse. Rambam Maimonides Med J 2017; 8: e0017.

7. Hallock JL, Handa VL. The epidemiology of pelvic floor disorders and childbirth: an update. Obster Gynecol Clin North Am 2016; 43: 1-13.

8. Staer-Jensen J, Siafarikas F, Hilde G, et al. Postpartum recovery of levator hiatus and bladder neck mobility in relation to pregnancy. Obstet Gynecol 2015; 125: 531-9.

9. Park YH, Yang SC, Park ST, et al. Laparoscopic reconstructive surgery is superior to vaginal reconstruction in the pelvic organ prolapse. Int J Med Sci 2014; 10: 1082-8.

10. Tailor D. The failure of polypropylene surgical mesh in vivo. J Mech Behav Biomed Mater 2018; 88: 370-6.

11. Barski D, Arndt C, Gerullis H, et al. Transvaginal PVDF-mesh for cystocele repair: a cohort study. Int J Surg 2017; 39: 249-54.

12. Di Biase M, Illiano E, Sarti E, et al. Polyvinyldenfluorid (PVDF) versus polypropylene (PP) mesh for sacrocolpopexy. J Urol 2017; 197: e354

13. Dällenbach P. To mesh or not to mesh: a review of pelvic organ reconstructive surgery. Int J Womens Health 2015; 7: 331-43.

14. Bissoli J, Bruschini H. Scaffolds for pelvic floor prolapse: logical pathways. Int J Biomater 2018; 2018: 8040893.

15. Barski D, Otto T, Gerullis H. Systematic review and classification of complications after anterior, posterior, apical, and tota vaginal mesh implantation for prolapse repair. Surg Technol Int 2014: $24: 217-24$
16. Shah HN, Badlani GH. Mesh complications in female pelvic floor reconstructive surgery and their management: a systematic review. Indian J Urol 2012; 28: 129-153.

17. Epstein M, Emri I, Hartemann P, et al. Opinion on the use safety of surgical meshes used in urogynecological surgery. SCENIHR - European Commission 2015; retrieved from https:// ec.europa.eu/health/scientific_committees/emerging/docs/ scenihr_o_049.pdf.

18. Chapple CH, Cruz F, Deffieux X, et al. Consensus statement of the European Urology Association and the European Urogynaecological Association on the use of implanted materials for treating pelvic organ prolapse and stress urinary incontinence. Eur Urol 2017; 72: 424-31.

19. Urogynecologic Surgical Mesh: Update on the Safety and Effectiveness of Transvaginal Placement for Pelvic Organ Prolapse. FDA 2011; https://www.fda.gov/downloads/medicaldevices/ safety/alertsandnotices/ucm262760.pdf.

20. Urogynecologic Surgical Mesh Implants. FDA 2019; https:// www.fda.gov/medical-devices/implants-and-prosthetics/urogynecologic-surgical-mesh-implants.

21. Surgical mesh implants - regulatory action on surgical mesh products. Medsafe 2018; https://www.medsafe.govt.nz/hot/ alerts/UrogynaecologicaSurgicalMeshImplants.asp.

22. Hijazi S, Echtle D, Aboumarzouk OM, et al. Abdominal sacrocolpopexy with Pelvicol xenograft and concomitant Burch colposuspension. Int J Womens Health 2017; 9: 625-30.

23. Dwyer PL. Choice of pelvic organ prolapse surgery: vaginal or abdominal, native tissue or synthetic grafts, open abdominal versus laparoscopic or robotic. Internat Urogynecol J 2014; 25: 1151-2.

24. Dietz, HP, Korda A. Which bowel symptoms are most strongly associated with a true rectocele? Aust N Z J Obstet Gynaecol 2005; 45: 505-8

25. Surkont G, Wlaźlak E, Stetkiewicz T, et al. Kolposakrofiksacja zmodyfikowanym sposobem Richtera - przebieg okresu okołoi pooperacyjnego pierwszych zabiegów. Menopause Rev 2005; 3: $65-71$.

26. Cespedes RD. Anterior approach bilateral sacrospinus ligament fixation for vaginal vault prolapse. Urology 2000; 56: 70-5.

27. Dietz, V, Huisman, M, de Jong JM, et al. Functional outcome after sacrospinous hysteropexy for uterine descensus. Int Urogynecol J Pelvic Floor Dysfunct 2008; 19: 747-52.

28. Nacheva A, Kovachev S, Markov V, et al. Richter's sacrospinous ligament fixation and its place in current gynecology. Akush Ginekol 2014; 53: 21-4.

29. Monk B, Ramp JL, Montz FJ, et al. Sacrospinus ligament fixation for vaginal vault prolapse. Complications and results. J Gynecol Surg 2009; 7: 87-92.

30. Jones $\mathrm{CH}$, Hatch KD, Harrigill K. Unilateral and bilateral sacrospinous ligament fixation for pelvic prolapse: a nonconcurrent cohort comparison. Fem Pelv Med Reconstr Surg 2001; 7: 27-33.

31. Lang P, Whiteside J. Anterior compartment prolapse - what's new? Curr Opin Obstet Gynecol 2017; 29: 337-42.

32. Velemir L., Amblard J, Fatton B, et al. Transvaginal mesh repair of anterior and posterior vaginal wall prolapse: a clinical and ultrasonographic study. Ultrasound Obstet Gynecol 2010; 35: 474-80. 
33. Comesu YM, Rogers GR, Kammerer-Doak DN, et al. Posterior repair and sexual function. Am J Obstet Gynecol 2007; 197101. e1-101-e6.

34. Ulubay M, Keskin U, Fidan U, et al. Safety, efficiency and outcomes of perineoplasty: treatment of the sensation of a wide vagina. Biomed Res Int 2016; 2016: 2495105.

35. Noé KG, Schiermeier S, Alkatout I, et al. Laparoscopic pectopexy: a prospective, randomized, comparative clinical trial of standard laparoscopic sacral colpocervicopexy with the new laparoscopic pectopexy - postoperative results and intermediate-term follow-up in a pilot study. J Endourol 2015; 29: 210-5.

36. Coolen AWM, van Oudheusden AMJ, Mol BWJ, et al. Laparoscopic sacrocolpopexy compared with open abdominal sacrocolpopexy for vault prolapse repair: a randomised controlled trial. Int Urogynecol J 2017; 28: 1469-79.

37. Kenneth G, Evers M, Terry CL, et al. Mesh erosion and abdominal sacrocolpopexy: a comparison of prior, total, and supracervical hysterectomy. J Pelvic Med Surg 2006; 12: 25-30.

38. Korbly NB, Kassis NC, Good MM, et al. Patient preferences for uterine preservation and hysterectomy in women with pelvic organ prolapse. Am J Obstet Gynecol 2013; 209: 470.e1-6.

39. Illiano E, Zucchi A, Giannitsas K, et al. Uterus preservin prolapse repair: how long does it last? Urol Int 2019; 102: 319-25.

40. Rahmanou P, White B, Price N, et al. Laparoscopic hysteropexy: 1 to 4-year follow-up of women postoperatively. Int Urogynecol J 2014; 25: 131-8.

41. Claerhout F, De Ridder D, Roovers JP, et al. Medium-term anatomic and functional results of laparoscopic sacrocolpopexy beyond the learning curve. Eur Urol 2009; 55: 1459-67.

42. Wong V, Guzman Rojas R, Shek KL, et al. Laparoscopic sacropexy: how low does the mesh go? Ultrasound Obstet Gynecol 2017; 49: 404-8.

43. Cosma S, Menato G, Ceccaroni M, et al. Laparoscopic sacropexy and obstructed defecation syndrome: an anatomoclinical study. Int Urogynecol I 2013; 24: 1623-30.

44. Deprest J, Krofta L, Van der AF, et al. The challenge of implementing laparoscopic sacrocolpopexy. Int Urogynecol I 2014; 25: 1153-60.

45. Manodoro S, Werbrouck E, Veldman J, et al. Laparoscopic sacrocolpopexy. Facts Views Vis Obgyn 2011; 3: 151-8.

46. Dubuisson J, Yaron M, Wenger JM, et al. Treatment of genital prolapse by laparoscopic lateral suspension using mesh: a series of 73 patients. J Minim Invasive Gynecol 2018; 15: 49-55.

47. Barber MD, Maher C. Apical prolapse. Int Urogynecol J 2013; 24 1815-30.

48. Dubuisson J, Veit-Rubin N, Bouquet de Jolinière J, et al. Laparoscopic lateral suspension: benefits of a cross-shaped mesh to treat difficult vaginal vault prolapse. I Minim Invasive Gynecol 2016; 23: 672.

49. Dubuisson J, Eperon I, Dällenbach P, et al. Laparoscopic repair of vaginal vault prolapse by lateral suspension with mesh. Arch Gynecol Obstet 2013; 287: 307-12.

50. Arenholt LTS, Pedersen BG, Glavind K, et al. Paravaginal defect: anatomy, clinical findings, and imaging. Int Urogynecol J 2017; 28: 661-73.

Received: 5.06.2019, accepted: 4.08.2019. 